\title{
Nonchaotic Behavior and Transition to Chaos in Lorenz-like Systems Having Invariant Algebraic Surfaces
}

\author{
Marcelo Messias (iD $*, 1$ and Rafael Paulino Silva (D) $*, 2$ \\ *Departamento de Matemática e Computação, Faculdade de Ciências e Tecnologia, Universidade Estadual Paulista (UNESP), São Paulo, Brazil.
}

ABSTRACT The famous and well-studied Lorenz system is considered a paradigm for chaotic behavior in three-dimensional continuous differential systems. After the appearance of such a system in 1963, several Lorenz-like chaotic systems have been proposed and studied in the related literature, as Rössler system, ChenUeta system, Rabinovich system, Rikitake system, among others. However, these systems are parameter dependent and are chaotic only for suitable combinations of parameter values. This raises the question of when such systems are not chaotic, which can be seen as a dual problem regarding chaotic systems. In this paper, we give sufficient algebraic conditions for a generalized class of Lorenz-like systems to be nonchaotic. Using the general results obtained, we give some examples of nonchaotic behavior of some classical "chaotic" Lorenz-like systems, including the Lorenz system itself. The nonchaotic differential systems presented here have invariant algebraic surfaces, which contain the stable (or unstable) invariant manifolds of their equilibrium points. We show that, in some cases, the deformation of these invariant manifolds through the destruction of the invariant algebraic surfaces, by perturbing the parameter values, can reorganize the global structure of the phase space, leading to a transition from nonchaotic to chaotic behavior of such differential systems.

\section{KEYWORDS}

Chaotic and nonchaotic dynamics Lorenz-like systems

Darboux theory of integrability Invariant algebraic surface

Darboux invariant

Stable and unstable manifolds.

\section{INTRODUCTION}

Let $\mathbb{R}[x, y, z]$ be the ring of polynomial functions in the variables $x, y, z$, with coefficients in $\mathbb{R}$. Consider the system of first order ordinary differential equations (or differential system for short) defined in $\mathbb{R}^{3}$ given by

$$
\dot{x}=P(x, y, z), \quad \dot{y}=Q(x, y, z), \quad \dot{z}=R(x, y, z),
$$

where $P, Q, R \in \mathbb{R}[x, y, z]$ and the dot denotes derivative with respect to the independent variable $t$, usually called the time, mainly in physical systems. The degree of system (1) is defined as the maximum of the degrees of polynomials $P, Q$ and $R$. When the maximum degree is two, system (1) is called quadratic.

Manuscript received: 11 November 2021,

Revised: 3 February 2022,

Accepted: 8 February 2022

1 marcelo.messias1@unesp.br (Corresponding Author)

2 paulino.silva@unesp.br
Beyond its theoretical importance, system (1) appears frequently in mathematical modeling of several dynamical phenomena arising in different areas, like Physics, Engineering, Biology and Chemistry, among others, as shown in the references (Alligood et al. 1996; Argyris et al. 2015; Cencini et al. 2010; Guckenheimer and Holmes 2002; Ott 2002; Strogatz 2001; Wiggins 2003). In this way, the study of the behavior of solutions of system (1) in its phase space is important to understand the phenomena modeled by it. The possible behaviors include stable and unstable equilibrium points and periodic orbits, quasi-periodic orbits, and chaotic dynamics. In particular, the interest in studying systems like (1) with chaotic behavior increased a lot in the last decades, due to their appearance in the study of several phenomena. One of the first chaotic systems studied was the famous and well-known Lorenz system (Lorenz 1963), which was the precursor of several other differential systems presenting such a behavior, like Rössler system (Rössler 1976), Rabinovich system (Llibre et al. 2008), Chen-Ueta system (Chen and Ueta 1999), Rikitake system (Llibre and Messias 2009), among others. The chaotic dynamics of these polynomial differential systems is directly related to the degree and values 
of coefficients (also called parameters) of the polynomials which determine them. In fact, although often called "chaotic systems" in the literature, the solutions of most of them present chaotic behavior only for certain combinations of their parameter values. For instance, it was shown by Edward Lorenz in 1963 that the solutions of system

$$
\begin{aligned}
& \dot{x}=s(y-x), \\
& \dot{y}=r x-y-x z, \\
& \dot{z}=-b z+x y,
\end{aligned}
$$

which has degree two (so it is quadratic), presents chaotic behavior if $s=10, b=\frac{8}{3}$ and $r=28$ (Lorenz 1963). Later on, it was shown by several authors that Lorenz system (2) has chaotic behavior for many other combinations of parameter values (see for instance the nice book (Sparrow 1982) or the more recent review (Algaba et al. 2018) and references cited in them).

The Lorenz system (2) is a polynomial differential system with peculiar quadratic nonlinearities, which appear in the second and third equations and are given by the crossed product of variables (i.e. $x z$ and $x y$ ). This motivated the definition of a more general class of quadratic differential systems called Lorenz-like systems, given by

$$
\begin{aligned}
& \dot{x}=a_{1} x+b_{1} y+c_{1} z+d_{1} y z, \\
& \dot{y}=a_{2} x+b_{2} y+c_{2} z+d_{2} x z, \\
& \dot{z}=a_{3} x+b_{3} y+c_{3} z+d_{3} x y,
\end{aligned}
$$

where $a_{i}, b_{i}, c_{i}, d_{i} \in \mathbb{R}, i=1,2,3$. This kind of system is often cited in the literature concerning chaotic systems, because several classical quadratic polynomial differential systems like Rössler, Rabinovich, Chen-Ueta, Rikitake, beyond the Lorenz system itself, can be obtained from system (3) by appropriate choice of the parameters $a_{i}, b_{i}, c_{i}, d_{i}$.

As the chaotic dynamics of several subclasses of system (3) were obtained in literature, a quite natural question arises in this context: can we determine conditions on the parameters of system (3) which can guarantee that it is nonchaotic? This is an important issue that can be seen as the dual problem of knowing when system (3) is chaotic. There are several papers dedicated to study the nonchaotic behavior of polynomial systems, especially the quadratic ones, see for instance the series of papers by Heidel and Zhang (Heidel and Zhang 1999, 2007; Zhang and Heidel 1997, 2012; Zhang et al. 2008), by Malasoma (Malasoma 2009, 2002) and Yang (Yang 2000, 2002; Yang and Chen 2002). The question about the nonchaotic behavior of differential systems is also related to the integrability theory (Dumortier et al. 2006; Llibre 2004; Llibre and Zhang 2012), because the phase space of integrable differential systems can be completely determined by their first integrals, hence they are not chaotic. Despite the existence of such studies, a general criterion for determining the nonchaotic behavior of polynomial differential systems defined in $\mathbb{R}^{3}$, or a general characterization of the $\omega$-limit sets of their solutions, like the Poincaré-Bendixson theorem for planar differential systems (Dumortier et al. 2006), is far from being obtained, even in the quadratic case.

In (Messias and Silva 2018), by using some elements of Darboux Theory of Integrability, namely invariant algebraic surfaces and Darboux invariants, we stated and proved a sufficient algebraic criterion which guarantees the nonchaotic behavior of differential system (1), for $P, Q, R$ polynomials of any degree. Using this criterion, we proved also in (Messias and Silva 2018) the nonchaotic behavior for a huge class of quadratic polynomial differential systems which have a symmetric Jacobian matrix, giving a partial answer for a conjecture proposed by Zeraoulia and Sprott, which states that "Three-dimensional quadratic continuous-time differential systems with a symmetric Jacobian matrix cannot be chaotic".

Later on, in (Messias and Silva 2020) we studied third order ordinary differential equations of the form

$$
\dddot{x}=j(x, \dot{x}, \ddot{x}),
$$

called jerk equations. When $j$ is a polynomial, it can be called a polynomial jerk equation. From the physical point of view, the third derivative can be seen as the derivative of the acceleration of a particle with position $x$, velocity $\dot{x}$ and acceleration $\ddot{x}$, so this type of equations has great interest in applications. Using the algebraic criterion stated in (Messias and Silva 2018), we obtained general conditions on the polynomial $j$ that guarantee the nonchaotic behavior of equation (4), which is equivalent to a subclass of system (1) by the natural change of coordinates $\dot{x}=y, \dot{y}=z, \dot{z}=j(x, y, z)$.

In the context above, in this paper our main goal is to determine sufficient conditions on the parameters which can guarantee the nonchaotic behavior of Lorenz-like system (3). The paper is organized as follows. In Section 2 we present some preliminary results from Darboux theory of integrability and use them to state a sufficient (but not necessary) algebraic criterion for the noncahotic behavior of system (1). Using this criterion, in Section 3 we state sufficient algebraic conditions for system (3) to be nonchaotic. In Section 4, we give some examples of classical systems derived from system (3) which we can guarantee that, for some combinations of parameter values, do not present chaotic behavior, as Lorenz system, Rabinovich system, Chen-Ueta system, and certain Lorenzlike systems with D2 symmetry (Anastassiou et al. 2002; Zhu C., Liu Y. and Guo Y. 2010). The nonchaotic differential systems presented here have invariant algebraic surfaces, which contain the stable or unstable invariant manifolds of their equilibrium points. In Section 5, we show that, in some cases, the deformation of these invariant manifolds through the destruction of the invariant algebraic surfaces, by perturbing the parameter values in system (3), can reorganize the global structure of the phase space, leading to a transition from nonchaotic to chaotic behavior of such differential systems. Finally, in Section 6 we present some concluding remarks and comments.

\section{SOME PRELIMINARIES FROM DARBOUX THEORY OF IN- TEGRABILITY}

Here, as in (Messias and Silva 2018), the existence of invariant algebraic surfaces and Darboux invariants are used to give a sufficient algebraic criterion which guarantees the nonchaotic behavior of three-dimensional polynomial differential systems (see Theorem 1 ahead). The definitions and results presented in this section also appear in (Messias and Silva 2018; Messias and Silva 2020) and in other classical texts about integrability theory (Dumortier et al. 2006; Llibre 2004; Llibre and Zhang 2012), but they are included here for the sake of completeness and to make the text easier to read.

The Darboux theory of integrability provides a link between the integrability of polynomial differential systems (or polynomial vector fields) and their invariant algebraic surfaces. A nice presentation of this theory for planar polynomial differential systems can be found in (Llibre 2004) and in Chapter 8 of (Dumortier et al. 2006). Here we are interested in quadratic polynomial differential systems defined in $\mathbb{R}^{3}$, hence we will present the results for system 
(1) with degree two, which is naturally associated to the vector field

$$
X(x, y, z)=P(x, y, z) \frac{\partial}{\partial x}+Q(x, y, z) \frac{\partial}{\partial y}+R(x, y, z) \frac{\partial}{\partial z},
$$

where $P, Q$ and $R$ are the polynomials in the right-hand side of system (1). In the next section we will apply the results in the study of the Lorenz-like system (3).

Definition 1 Let $U$ be an open subset of $\mathbb{R}^{3}$. If there exists a nonlocally constant differentiable function $H: U \rightarrow \mathbb{R}$, which is constant on all solution curves $(x(t), y(t), z(t))$ of system (1) (or of the vector field (5)) contained in $U$, then $H$ is called a first integral of $X$ in $U$. Clearly $H$ is a first integral of system (1) if and only if $X(H) \equiv 0$ on $U$, i.e.

$$
X(H)=\frac{d H}{d t}=\frac{\partial H}{\partial x} P+\frac{\partial H}{\partial y} Q+\frac{\partial H}{\partial z} R=0
$$

on the orbits of $X$ contained in $U$, where $H=H(x(t), y(t), z(t))$.

Definition 2 An invariant of system (1) on an open subset $U \subset \mathbb{R}^{3}$ is a nonlocally constant differentiable function I in the variables $x, y, z$ and $t$ such that $I$ is constant on all solution curves $(x(t), y(t), z(t))$ of system (1) contained in $U$, i.e.

$$
\frac{d I}{d t}=\frac{\partial I}{\partial x} P+\frac{\partial I}{\partial y} Q+\frac{\partial I}{\partial z} R+\frac{\partial I}{\partial t}=0
$$

on the orbits of $X$ contained in $U$.

An invariant $I$ can be seen as a first integral of system (1) which depends on the time $t$.

Definition 3 Let $f \in \mathbb{K}[x, y, z]$ be a non-locally constant polynomial, where $\mathbb{K}$ is either $\mathbb{R}$ or $\mathbb{C}$. The surface $f(x, y, z)=0$ is an invariant algebraic surface of system (1) if there exists a polynomial $K \in \mathbb{K}[x, y, z]$ such that

$$
X(f)=\frac{\partial f}{\partial x} P+\frac{\partial f}{\partial y} Q+\frac{\partial f}{\partial z} R=K f .
$$

The polynomial $K$ is called the cofactor of the invariant algebraic surface $f=0$.

Note that, as system (1) has degree 2, then the degree of the cofactor $K$ is at most 1 . Moreover, when $K=0$, then $f$ is a polynomial first integral of system (1).

Definition 4 Let $g, h \in \mathbb{K}[x, y, z] \backslash\{0\}$ and assume that $g$ and $h$ are relatively prime polynomials in the ring $\mathbb{K}[x, y, z]$, or that $h=1$, where $\mathbb{K}$ is either $\mathbb{R}$ or $\mathbb{C}$. Then the function $F=\exp (\mathrm{g} / \mathrm{h})$ is called an exponential factor of system (1) if for some polynomial $L \in \mathbb{K}[x, y, z]$ of degree at most $m-1$ we have that

$$
X(F)=\frac{\partial F}{\partial x} P+\frac{\partial F}{\partial y} Q+\frac{\partial F}{\partial z} R=L F .
$$

We say that an invariant $I$ of $X$ is of Darboux type or a Darboux invariant if it can be written as

$$
I(x, y, z, t)=f_{1}^{\lambda_{1}} \ldots f_{p}^{\lambda_{p}} F_{1}^{\mu_{1}} \ldots F_{q}^{\mu_{q}} e^{s t},
$$

where $f_{i}=0$ are invariant algebraic surfaces of $X$ for $i=1, \ldots, p$; $F_{j}$ are exponential factors of $X$ for $j=1, \ldots, q ; \lambda_{i}, \mu_{j} \in \mathbb{C}$ and $s \in \mathbb{R} \backslash\{0\}$.

The following result holds.

Proposition 1 If $f(x, y, z)=0$ is an invariant algebraic surface of system (1) with a constant cofactor $K=k \in \mathbb{R} \backslash\{0\}$, then $I=$ $f(x, y, z) e^{-k t}$ is a Darboux invariant of this system.
Proof. Let $\phi(t)=(x(t), y(t), z(t))$ be a solution and $f=0$ be an invariant algebraic surface of system (1) (or of the vector field (5)). Then we have

$$
\begin{aligned}
\frac{d}{d t} I(\phi(t)) & =\frac{d}{d t}\left[f(\phi(t)) e^{-k t}\right]= \\
& =\left[\frac{\partial f}{\partial x} P+\frac{\partial f}{\partial y} Q+\frac{\partial f}{\partial z} R\right] e^{-k t}-k f(\phi(t)) e^{-k t}= \\
& =k f(\phi(t)) e^{-k t}-k f(\phi(t)) e^{-k t}=0
\end{aligned}
$$

While the knowledge of a first integral of system (1) in $\mathbb{R}^{3}$ allows to reduce its study in one dimension, the knowledge of a Darboux invariant provides information about the $\alpha$ and $\omega$-limit sets of all orbits of system (1). Indeed, the following result, proved in (Llibre and Oliveira 2015) for planar polynomial differential systems, can be easily extended to polynomial differential systems defined in $\mathbb{R}^{3}$ and gives a relation between the existence of Darboux invariants and the $\alpha$ and $\omega$-limit sets of the solutions of such systems.

Proposition 2 Let $I(x, y, z, t)=f(x, y, z) e^{\text {st }}$ be a Darboux invariant of system (1). Let $p \in \mathbb{R}^{3}$ and $\varphi_{p}(t)$ be the solution of system (1) with maximal interval $\left(\alpha_{p}, \omega_{p}\right)$ such that $\varphi_{p}(0)=p$. The following statements hold.

(a) If $\omega_{p}=\infty$ then $\omega(p) \subset \overline{\{f(x, y, z)=0\}} \cup \mathbb{S}^{2}$, where $\mathbb{S}^{2}$ is the boundary of the Poincaré ball (at infinity).

(b) If $\alpha_{p}=-\infty$ then $\alpha(p) \subset \overline{\{f(x, y, z)=0\}} \cup S^{2}$, where $S^{2}$ is the boundary of the Poincaré ball (at infinity).

The definition of Poincaré ball is given, for instance, in (Llibre et al. 2008). Note that in Proposition 2 the function $f$ is of the form $f=f_{1}^{\lambda_{1}} \ldots f_{p}^{\lambda_{p}} F_{1}^{\mu_{1}} \ldots F_{q}^{\mu_{q}}$, as in (6).

In (Messias and Silva 2018) we proved the following result.

Theorem 1 [Algebraic criterion for nonchaoticity] Let $X$ be the vector field (5), associated to differential system (1). If $X$ has an invariant algebraic surface $f=0$ with a constant cofactor $K=k \in \mathbb{R} \backslash\{0\}$, then the $\alpha$ and $\omega$-limit sets of each orbit $\phi_{p}(t)=(x(t), y(t), z(t))$ with $\phi_{p}(0)=p \in \mathbb{R}^{3}$, are both contained in $\overline{\{f=0\}} \cup S^{2}$, where $S^{2}$ represents the points at infinity of $\mathbb{R}^{3}$. In particular, $X$ does not present chaotic behavior.

The algebraic criterion stated in Theorem 1 gives a sufficient but not necessary condition for the nonchaotic behavior of the vector field (5). Indeed, there are several differential systems proved to be nonchaotic in the literature (Zhang and Heidel 1997, 2012; Zhang et al. 2008; Yang 2000, 2002; Yang and Chen 2002), which have no invariant algebraic surfaces. Furthermore, we observe that the hypothesis of a constant cofactor is essential in Theorem 1. Indeed, in (Jafari et al. 2016; Li et al. 2021) the authors gave examples of chaotic systems which have algebraic surfaces formed by equilibrium points (which are obviously invariant algebraic surfaces). However, we checked these cases and in all of them the cofactors of the invariant algebraic surfaces are not constant.

In the next section we will use Theorem 1 to obtain sufficient conditions for the nonchaotic behavior of Lorenz-like systems (3). 


\section{STATEMENT AND PROOF OF THE MAIN RESULTS: NON- CHAOTIC LORENZ-LIKE SYSTEMS}

In the following result we give a huge class of Lorenz-like systems which do not present chaotic behavior.

Theorem 2 Let $X=X(x, y, z)$ be the vector field associated to system (3). For the parameter values $a_{1}=b_{2}=\frac{1}{2} k, c_{3}=k, d_{3} \neq 0$ and the other parameters satisfying the system

$$
\left\{\begin{array}{c}
\left(2 a_{2} c_{1}+k c_{2}\right) d_{3}-2 a_{3}\left(a_{2} d_{1}-b_{1} d_{2}\right)=0, \\
\left(2 b_{1} c_{2}+k c_{1}\right)+2 b_{3}\left(a_{2} d_{1}-b_{1} d_{2}\right)=0
\end{array}\right.
$$

the vector field $X=X(x, y, z)$ has the invariant algebraic surface

$$
f(x, y, z)=-d_{2} x^{2}-2 c_{2} x+d_{1} y^{2}+2 c_{1} y-2 \frac{a_{2} d_{1}-b_{1} d_{2}}{d_{3}} z
$$

with cofactor $k \in \mathbb{R}$. Consequently, for these choice of parameters, system (3) does not present chaotic behavior.

Proof. Consider the vector field $X=(P, Q, R)$ associated to system (3) with the choice of parameters given in Theorem 2 . Then, the system reduces to

$$
\begin{aligned}
& \dot{x}=P(x, y, z)=\frac{k}{2} x+b_{1} y+c_{1} z+d_{1} y z, \\
& \dot{y}=Q(x, y, z)=a_{2} x+\frac{k}{2} y+c_{2} z+d_{2} x z, \\
& \dot{z}=R(x, y, z)=a_{3} x+b_{3} y+k z+d_{3} x y,
\end{aligned}
$$

where the parameters $a_{2}, a_{3}, b_{1}, b_{3}, c_{1}, c_{2}, d_{1}, d_{2}$ satisfy system (7). In this way, the function (8) is a Darboux polynomial of system (9), with cofactor $k \in \mathbb{R}$. In fact, we have

$$
\begin{aligned}
& \langle\nabla f, X\rangle= \\
& \frac{\partial f}{\partial x}(x, y, z) P(x, y, z)+\frac{\partial f}{\partial y}(x, y, z) Q(x, y, z)+\frac{\partial f}{\partial z}(x, y, z) R(x, y, z) \\
& =-k d_{2} x^{2}-k c_{2} x+2 c_{1} a_{2} x+k d_{1} y^{2}+k c_{1} y-2 c_{2} b_{1} y- \\
& -2 k \frac{\left(a_{2} d_{1}-b_{1} d_{2}\right)}{d_{3}} z-2 a_{3} \frac{\left(a_{2} d_{1}-b_{1} d_{2}\right)}{d_{3}} x-2 b_{3} \frac{\left(a_{2} d_{1}-b_{1} d_{2}\right)}{d_{3}} y \\
& =k\left(-d_{2} x^{2}-2 c_{2} x+d_{1} y^{2}+2 c_{1} y-2 k \frac{\left(a_{2} d_{1}-b_{1} d_{2}\right)}{d_{3}} z\right) \\
& +\frac{\left(2 a_{2} c_{1}+k c_{2}\right) d_{3}-2 a_{3}\left(a_{2} d_{1}-b_{1} d_{2}\right)}{d_{3}} x- \\
& -\frac{\left(2 b_{1} c_{2}+k c_{1}\right)+2 b_{3}\left(a_{2} d_{1}-b_{1} d_{2}\right)}{d_{3}} y \\
& =k f(x, y, z) .
\end{aligned}
$$

Hence, $f(x, y, z)=0$ is an invariant algebraic surface of system (3), with cofactor $k \in \mathbb{R}$. Therefore, by Theorem 1 this system does not present chaotic behavior.

Remark Let $X=X(x, y, z)$ be the vector field associated to system (9) and $f(x, y, z)=0$ the invariant algebraic surface given in Theorem 2. Then, we have that

a) $f(x, y, z)$ is a first integral of system (9) if, and only if, $k=0$. In this case, the phase space is foliated by the invariant algebraic surfaces $f(x, y, z)=c, c \in \mathbb{R}$;

b) The vector field $X$ is dissipative if, and only if, $k<0$. If $k=0$, then $X$ is conservative.
Theorem 3 Let $X=X(x, y, z)$ be the vector field associated to system (3) with the choice of parameters $a_{1}=b_{2}=c_{3}=\frac{1}{2} k, b_{1}=a_{2} \alpha$, and

$$
\left\{\begin{array}{c}
c_{1} d_{3}+a_{3}\left(d_{2} \alpha-d_{1}\right)=0, \\
\alpha c_{2} d_{3}-b_{3}\left(d_{2} \alpha-d_{1}\right)=0,
\end{array}\right.
$$

where $\alpha, k \in \mathbb{R}$ and $\alpha d_{3} \neq 0$. Then, system (3) presents the invariant algebraic surface

$$
f(x, y, z)=-x^{2}+\alpha y^{2}-\frac{\left(d_{2} \alpha-d_{1}\right)}{d_{3}} z^{2},
$$

with cofactor $k \in \mathbb{R}$. Consequently, the system does not present chaotic behavior.

Proof. Consider the vector field $X=(P, Q, R)$ associated to system (3) with the choice of parameters given in Theorem 3 . Then, we have

$$
\begin{aligned}
& \dot{x}=P(x, y, z)=\frac{k}{2} x+a_{2} \alpha y+c_{1} z+d_{1} y z, \\
& \dot{y}=Q(x, y, z)=a_{2} x+\frac{k}{2} y+c_{2} z+d_{2} x z, \\
& \dot{z}=R(x, y, z)=a_{3} x+b_{3} y+\frac{k}{2} z+d_{3} x y .
\end{aligned}
$$

In this way, the function (11) is a Darboux polynomial of system (12) with cofactor $k \in \mathbb{R}$. In fact, we have

$$
\begin{aligned}
& \langle\nabla f, X\rangle= \\
& =\frac{\partial f}{\partial x}(x, y, z) P(x, y, z)+\frac{\partial f}{\partial y}(x, y, z) Q(x, y, z)+\frac{\partial f}{\partial z}(x, y, z) R(x, y, z)= \\
& =-k x^{2}+k \alpha y^{2}-k \frac{\left(d_{2} \alpha-d_{1}\right)}{d_{3}} z^{2}-2 c_{1} x z+2 \alpha c_{2} y z \\
& -2 a_{3} \frac{\left(d_{2} \alpha-d_{1}\right)}{d_{3}} x z-2 b_{3} \frac{\left(d_{2} \alpha-d_{1}\right)}{d_{3}} y z \\
& =k\left(-x^{2}+\alpha y^{2}-\frac{\left(d_{2} \alpha-d_{1}\right)}{d_{3}} z^{2}\right) \\
& -2 \frac{\left(c_{1} d_{3}+a_{3}\left(d_{2} \alpha-d_{1}\right)\right)}{d_{3}} x z+2 \frac{\left(\alpha c_{2} d_{3}-b_{3}\left(d_{2} \alpha-d_{1}\right)\right)}{d_{3}} y z \\
& =k f(x, y, z) .
\end{aligned}
$$

Hence, $f(x, y, z)=0$ is an invariant algebraic surface of system (3), with cofactor $k \in \mathbb{R}$. Therefore, by Theorem 1 this system does not present chaotic behavior.

It follows from the results above that system (3) has a plane as an invariant algebraic surface if and only if $d_{1}=d_{2}=d_{3}=0$, that is, only when it is linear. On the other hand, such system may have invariant algebraic surfaces with degrees $n \geq 3$, however we did not study these cases in this note, since the conditions on the coefficients are very huge and complicated, due to the great number of parameters.

\section{EXAMPLES AND APPLICATIONS}

We can relate the classes of nonchaotic systems obtained in Theorems 2 and 3 with some classical Lorenz-like systems which appear in the literature. Since some "chaotic" Lorenz-like systems are actually chaotic only for some parameter values, they can be nonchaotic for other choices of parameters. Some examples are presented below. 


\section{Lorenz system}

The Lorenz systems (2) is maybe the most famous system which is known to be chaotic for certain parameter values (Algaba et al. 2018; Lorenz 1963; Sparrow 1982). Using Theorem 2 we can obtain some subclasses of Lorenz system which are not chaotic. We observe that a more general and detailed study of Lorenz system having invariant algebraic surfaces, including the dynamics at infinity via the Poincaré compactification, was made in (Llibre et al. 2010)

a) Consider in Theorem 2, the parameter values $a_{3}=b_{3}=c_{1}=$ $c_{2}=d_{1}=0, b_{1}=d_{3}=1, d_{2}=-1, k=-2$ and $a_{2} \in \mathbb{R}$, which corresponds to the Lorenz system (2) with parameters $s=1, r=a_{2}$ and $b=2$, that is

$$
\begin{aligned}
& \dot{x}=y-x, \\
& \dot{y}=a_{2} x-y-x z, \\
& \dot{z}=-2 z+x y,
\end{aligned}
$$

which has the parabolic cylinder $x^{2}-2 z=0$ as an invariant algebraic surface with cofactor $k=-2$, hence from Theorem 2 , it is not chaotic.

b) Consider the choice of parameters in Theorem 3 as $a_{3}=b_{3}=$ $c_{1}=c_{2}=d_{1}=0, d_{2}=-1, d_{3}=1, \alpha=\frac{1}{a_{2}}, k=-2$, and $a_{2} \neq 0$, which corresponds to the Lorenz system (2) with parameters $s=$ $1, r=a_{2}$ and $b=1$. Then, we get the nonchaotic system

$$
\begin{aligned}
& \dot{x}=y-x, \\
& \dot{y}=a_{2} x-y-x z, \\
& \dot{z}=-z+x y,
\end{aligned}
$$

which has the cone $-x^{2}+\frac{1}{a_{2}}\left(y^{2}+z^{2}\right)=0$ as an invariant algebraic surface with cofactor $k=-2$.

The phase portraits of systems (13) and (14) on the respective invariant algebraic surfaces are shown in Figures 1 and 2.
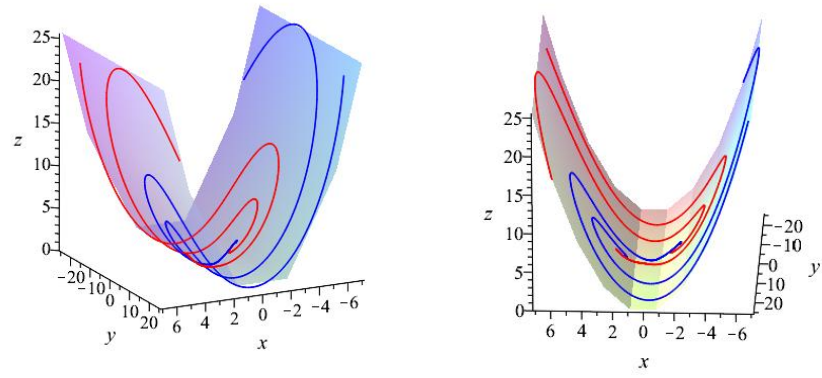

Figure 1 Phase portrait of system (13) with $a_{2}=3$.
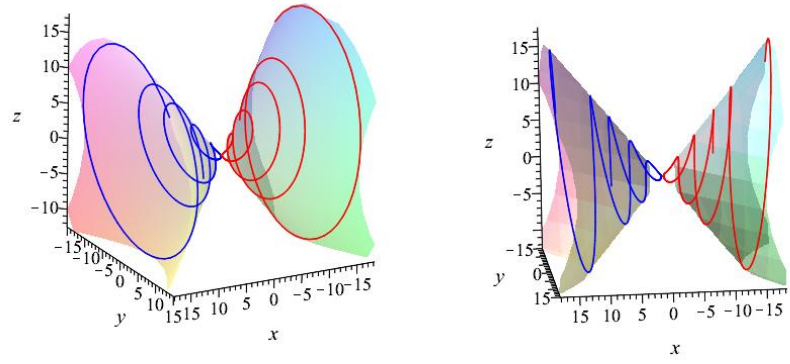

Figure 2 Phase portrait of system (14) with $a_{2}=1$.

\section{Rabinovich system}

The Rabinovich system (Llibre et al. 2008) is an example of Lorenzlike system. It is given by

$$
\begin{aligned}
& \dot{x}=h y-v_{1} x+y z, \\
& \dot{y}=h x-v_{2} y-x z, \\
& \dot{z}=-v_{3} z+x y,
\end{aligned}
$$

with $h, v_{1}, v_{2}, v_{3} \in \mathbb{R}$. It is known that system (15) presents chaotic behavior for the parameter values $v_{1}=4, v_{2}=v_{3}=1$ and $h=6,75$ (Llibre et al. 2008). Using Theorem 2, we can obtain the following cases in which the Rabinovich system has an invariant algebraic surface with constant cofactor, thus the system does not present chaotic behavior in theses cases.

a) Following Theorem 3 and considering the parameters $a_{3}=b_{3}=$ $c_{1}=c_{2}=0, d_{1}=d_{3}=1, d_{2}=-1, b_{1}=a_{2}$, and $k=-2 v$ in system (15), we obtain $v_{1}=v_{2}=v, v_{3}=2 v$ with $v \in \mathbb{R}$ and $h=a_{2}$, which lead to the following system

$$
\begin{aligned}
& \dot{x}=h y-v x+y z, \\
& \dot{y}=h x-v y-x z, \\
& \dot{z}=-2 v z+x y,
\end{aligned}
$$

which has the invariant algebraic surface $x^{2}+y^{2}-4 h z=0$ with constant cofactor $-2 v \in \mathbb{R}$. From Theorem 3 follows that system (16) is not chaotic.

b) Following Theorem 3 and considering the parameters $a_{3}=b_{3}=$ $c_{1}=c_{2}=0, d_{1}=d_{3}=1, d_{2}=-1, \alpha=1$ and $k=-2 v$ with $v \in \mathbb{R}$ in system (15), we obtain $v_{1}=v_{2}=v_{3}=v, h=a_{2}$ and the system

$$
\begin{aligned}
& \dot{x}=h y-v x+y z, \\
& \dot{y}=h x-v y-x z, \\
& \dot{z}=-v z+x y,
\end{aligned}
$$

which has the invariant algebraic surface $-x^{2}+y^{2}+2 z^{2}=0$, with constant cofactor $-2 v \in \mathbb{R}$, therefore it is not chaotic.

The phase portraits of systems (16) and (17) on the invariant algebraic surfaces described above are shown in Figures 3 (a) and (b), respectively. 


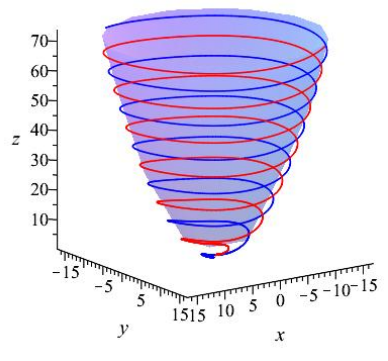

(a) $h=v=1$

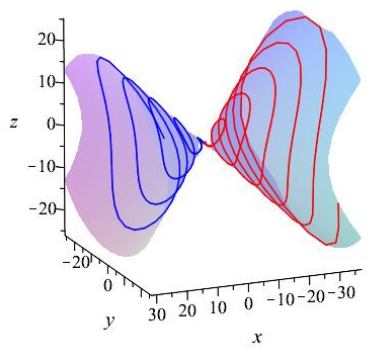

(b) $h=v=1$
Figure 3 (a) Phase portrait of system (16); (b) Phase portrait of system (17). In both cases, $v=h=1$.

\section{Chen-Ueta system}

As a subclass of the Lorenz-like system (3) there is the known Chen-Ueta system, given by (Chen and Ueta 1999)

$$
\begin{aligned}
& \dot{x}=a(y-x), \\
& \dot{y}=(c-a) x+c y-x z, \\
& \dot{z}=-b z+x y,
\end{aligned}
$$

where $a, b, c \in \mathbb{R}$. System (18) has chaotic behavior for the parameter values $a=35, b=3$ and $c=28$, as shown in (Chen and Ueta 1999). The global dynamical behavior of system (18) having invariant algebraic surfaces, including the behavior at infinity using Poincaré compactification, was done in (Llibre et al. 2012). Using Theorem 2, we can give some examples of Chen-Ueta system without chaotic behavior.

Considering Theorem 2 and taking the parameters $a_{3}=b_{3}=$ $c_{1}=c_{2}=d_{1}=0, a_{2}=k, b_{1}=-\frac{1}{2} k, d_{2}=-1, d_{3}=1, c_{2}=0, k=$ $2 r$ with $r \in \mathbb{R}$ in system (18) we obtain $a=-r, c=r, b=-2 r$ and the nonchaotic system

$$
\begin{aligned}
& \dot{x}=-r(y-x), \\
& \dot{y}=2 r x+r y+x z, \\
& \dot{z}=2 r z-x y,
\end{aligned}
$$

which has $x^{2}-2 r z=0$ as invariant algebraic surface, with constant cofactor $k=2 r$. The phase portrait of system (19) on this surface is given in Figure 4.

\section{Lorenz-like system with $D 2$ Symmetry}

In (Anastassiou et al. 2002), the authors studied the following differential system, derived from Lorenz-like system (3):

$$
\begin{aligned}
& \dot{x}=a_{1} x+d_{1} y z, \\
& \dot{y}=b_{2} y+d_{2} x z, \\
& \dot{z}=c_{3} z+d_{3} x y,
\end{aligned}
$$

where $a_{1}, b_{2}, c_{3}, d_{1}, d_{2}, d_{3} \in \mathbb{R}$. System (20) has several types of symmetry, as pointed out in (Anastassiou et al. 2002), and has
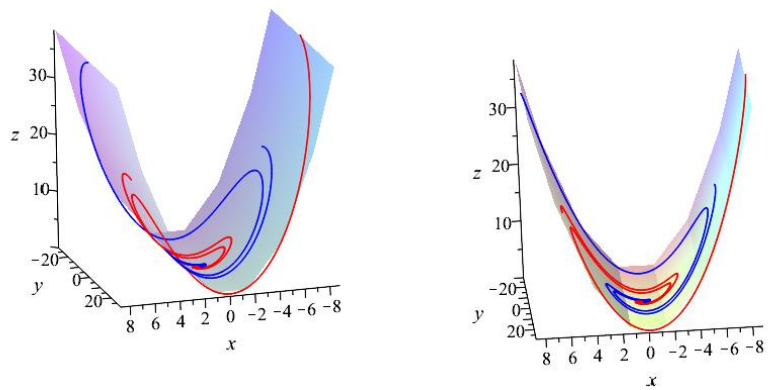

Figure 4 Phase portrait of Chen system (19) with $r=1$.

chaotic behavior for some choices of parameter values $a_{1}$ and $b_{2}$. They also showed that the function $V(x, y, z)=x^{2}+y^{2}+2 z^{2}$ is a Lyapunov function for system (20) if $a_{1}, b_{2}>0$ and $c_{3}=d_{1}=$ $d_{2}=1, d_{3}= \pm 1$.

Considering in Theorem 3 the parameter values $a_{2}=a_{3}=$ $b_{3}=c_{1}=c_{2}=0$ and taking $a_{1}=b_{2}=c_{3}=k / 2$ we obtain the following subclass of system (20)

$$
\begin{aligned}
& \dot{x}=\frac{k}{2} x+d_{1} y z, \\
& \dot{y}=\frac{k}{2} y+d_{2} x z, \\
& \dot{z}=\frac{k}{2} z+d_{3} x y .
\end{aligned}
$$

From Theorem 3, it follows that system (20) has no chaotic dynamics in this case, for any parameters $d_{1}, d_{2}, d_{3} \in \mathbb{R}$, with $d_{3} \neq 0$, since it has the invariant algebraic surface

$$
f(x, y, z)=-x^{2}+\alpha y^{2}-\frac{\left(d_{2} \alpha-d_{1}\right)}{d_{3}} z^{2}=0,
$$

with constant cofactor $k \in \mathbb{R}$, for any $\alpha \in \mathbb{R}$.

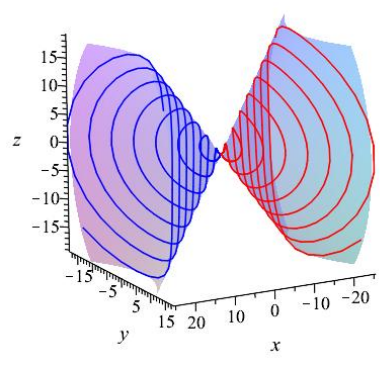

(a) $\alpha=2$

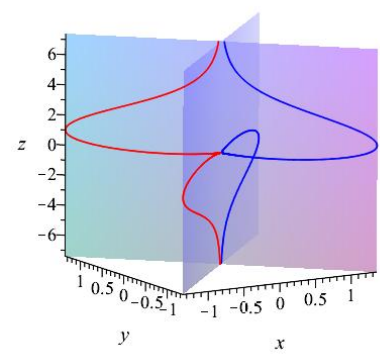

(b) $\alpha=1$
Figure 5 Phase portrait of $D 2$ system in the case of system (21) for the parameters $k=2, d_{1}=d_{2}=1, d_{3}=-1$, and: (a) $\alpha=2$; (b) $\alpha=1$.

From these calculations, we can see that for the choice of parameters $c_{1}=d_{1}=d_{2}=1, d_{3}=-1$, system (20) has a cone and two planes intersecting at the $z$-axis as invariant algebraic surfaces. In fact, taking $\alpha=1$, we obtain from equation (22) that $-x^{2}+y^{2}=0$ is an invariant algebraic surface with cofactor $k$. Furthermore, for $\alpha=2$ we obtain $-x^{2}+2 y^{2}+z^{2}=0$, which implies that system (20) has an invariant cone (see Figure 5 (a)), and for $\alpha=1$ this system has two invariant planes, see Figure 5 (b) ). These results complement the ones obtained in (Anastassiou et al. 2002). 


\section{Zhu-Liu-Guo symmetric Lorenz-like system}

In (Zhu C., Liu Y. and Guo Y. 2010), the authors studied the Lorenzlike system given by

$$
\begin{aligned}
& \dot{x}=-x-\beta_{1} y+y z, \\
& \dot{y}=\beta_{2} y-x z, \\
& \dot{z}=-\beta_{3} z+x y,
\end{aligned}
$$

where $\beta_{1}, \beta_{2}, \beta_{3} \in \mathbb{R}$. This system has the symmetry $(x, y, z) \mapsto$ $(-x,-y, z)$ and present chaotic behavior for certain parameter values, as shown in (Zhu C., Liu Y. and Guo Y. 2010). Considering Theorem 2, and choosing $a_{2}=a_{3}=b_{3}=c_{1}=c_{2}=0, d_{1}=d_{3}=$ $1, d_{2}=-1$, with $b_{1} \neq 0$ and $k=-2$, we obtain $\beta_{1}=-b_{1}, \beta_{2}=$ $-1, \beta_{3}=2$ and the subclass of system (23) given by

$$
\begin{aligned}
& \dot{x}=-x-b_{1} y+y z, \\
& \dot{y}=-y-x z, \\
& \dot{z}=-2 z+x y,
\end{aligned}
$$

which has the invariant algebraic surface $x^{2}+y^{2}+2 b_{1} z=0$, with cofactor $k=-2$. It follows from Theorem 1 that system (24) do not present chaotic behavior, see their phase portrait on the respective invariant algebraic surface in Figure 6.

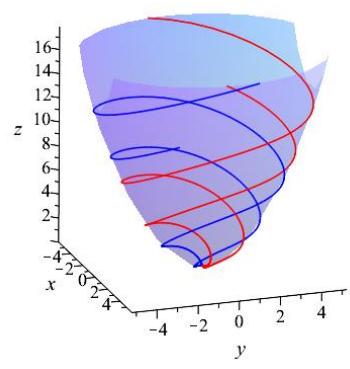

Figure 6 Phase portrait of Zhu system (24), with $b_{1}=-1$.

\section{TRANSITION FROM NONCHAOTIC TO CHAOTIC BEHAVIOR IN LORENZ-LIKE SYSTEMS}

In this section we will study the transition from nonchaotic to chaotic behavior in some Lorenz-like systems. The nonchaotic differential systems presented in the previous sections have invariant algebraic surfaces with constant cofactor, hence their equilibrium points are contained in the invariant algebraic surfaces, which therefore contain the stable (or unstable) manifolds of these equilibria. We will see that a small perturbation in the parameters of a nonchaotic system can destroy the invariant algebraic surface and, consequently, deform the invariant manifolds and reorganize the global structure of the phase space, leading to the creation of chaotic behavior in these systems. In order to show this transition from nonchaotic to chaotic behavior, via perturbation, we will analyze the Rabinovich system (15). As already mentioned, this system presents chaotic behavior for the parameter values $v_{1}=4, v_{2}=v_{3}=1$ and $h=6.75$, having in this case the chaotic attractor shown in Figure 7.

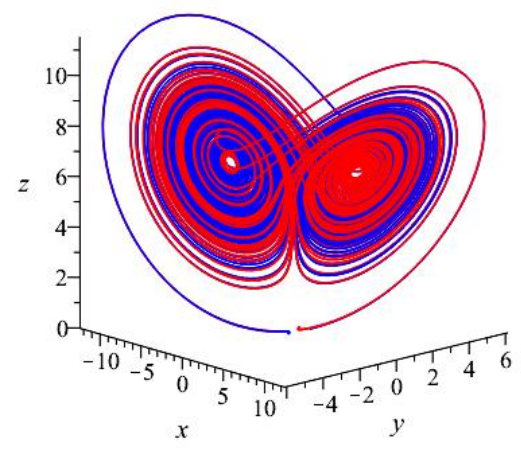

Figure 7 Chaotic attractor of Rabinovich system (15) with parameter values $h=6.75, v_{1}=4, v_{2}=v_{3}=1$.

Considering now system (15) in the conditions of Theorem 3, taking the parameter values $h=a_{2}, v_{1}=v_{2}=v_{3}=v$, we obtain system (17), which presents the cone $-x^{2}+y^{2}+2 z^{2}=0$ as an invariant algebraic surface with cofactor $k=-2 v \in \mathbb{R}$, hence it has no chaotic behavior (see Figure 3 (b)). In order to destroy the invariant algebraic surface in such a way that system (17) can generate chaotic behavior, we will use the following variation of this system

$$
\begin{aligned}
& \dot{x}=h y-v_{1} x+y z \\
& \dot{y}=h x-v y-x z \\
& \dot{z}=-v z+x y
\end{aligned}
$$

where $v_{2}=v_{3}=v$ are fixed and equal to 1 and $h=6.75$. Then, we will vary $v_{1}$ in order to deform the invariant cone and produce chaotic behavior. Varying $v_{1}$ in the interval $[1,3.8]$, we obtained the solutions of system (25) with initial conditions $( \pm 0.6, \pm 0.6,0)$, shown in Figures 8 to 12. In these figures, we can see the deformation of the invariant cone and the transition of solutions ranging from nonchaotic to chaotic behavior.

We observe that, when the parameter $v_{1}=1$, system (25) presents a cone as an invariant algebraic surface and three singular points belonging to the invariant cone: a saddle at the origin and two stable foci. Hence, the stable manifolds of the foci are contained in the invariant cone. When $v_{1}$ is different from 1 , the structure given by the invariant cone and the singular points is deformed and the system has no longer invariant algebraic surfaces, so the invariant manifolds are deformed. As $v_{1}$ moves away from $v_{1}=1$, the behavior of solutions become more and more complex and, for $v_{1}=3.8$ the chaotic attractor is created, as shown in Figure 12.

We can conclude that the formation of chaotic dynamics was due to the deformation of the invariant manifolds of the equilibrium points, which were initially (for $v_{1}=1$ ) contained on the invariant cone, with the destruction of this cone (for $v_{1} \neq 1$ ).

The same type of analysis can be done for the Rabinovich chaotic system presented in (Llibre et al. 2008). In such work, the authors have shown that system (15) presents a four-wings chaotic attractor for the parameter values given by $h=0.04, v_{1}=$ $-1.5, v_{2}=-0.3$ and $v_{3}=-1.67$, as shown in see Figure 13. Let us see that this chaotic attractor can be obtained by the deformation of an invariant algebraic surface through the variation of the parameter values. 


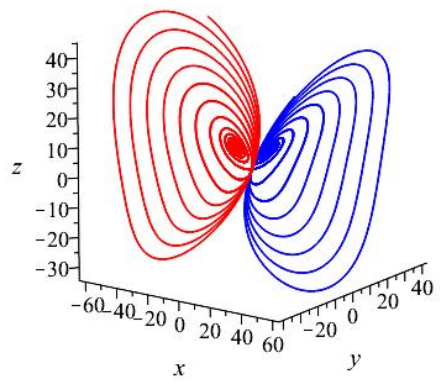

Figure 8 Two solutions of Rabinovich system (25) with parameter values $h=6.75, v=v_{1}=1$ and initial conditions $( \pm 0.6, \pm 0.6,0)$.

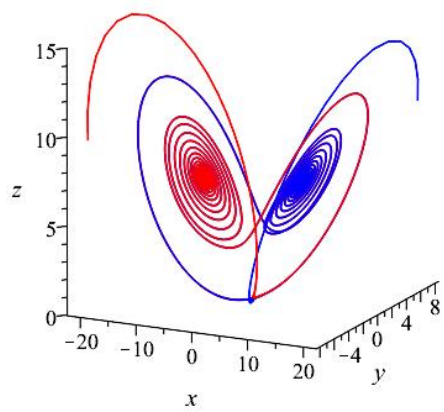

Figure 9 Two solutions of Rabinovich system (25) with parameter values $h=6.75, v=1, v_{1}=1.5$ and initial conditions $( \pm 0.6, \pm 0.6,0)$.

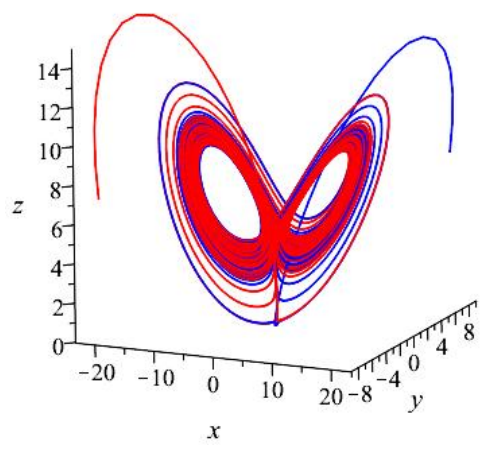

Figure 10 Two solutions of Rabinovich system (25) with parameter values $h=6.75, v=1, v_{1}=2$ and initial conditions $( \pm 0.6, \pm 0.6,0)$.

Consider the Rabinovich system (15) with $h=0.0, v_{1}=v_{3}$ and $v_{2} \in \mathbb{R}$. In this case, system (15) is in the hypothesis of Theorem 3 , having the invariant algebraic surface $-x^{2}+z^{2}=0$, given by two intersecting invariant planes, with constant cofactor $-2 v_{1}$, hence it does not present chaotic behavior. In order to study the deformation of the invariant planes of system (15), we will vary the parameter $h$ in the interval $[0,0.0201]$ and consider also $v_{1} \neq v_{3}$.

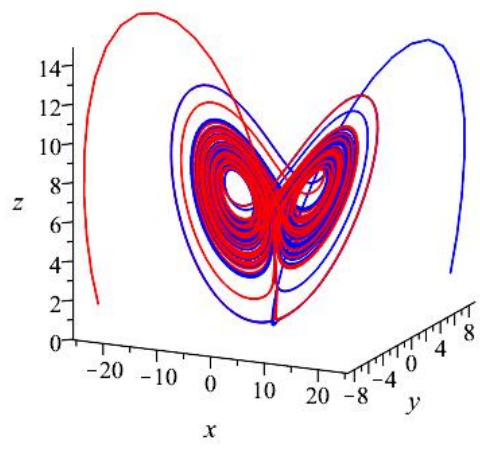

Figure 11 Two solutions of Rabinovich system (25) with parameter values $h=6.75, v=1, v_{1}=3$ and initial conditions $( \pm 0.6, \pm 0.6,0)$.

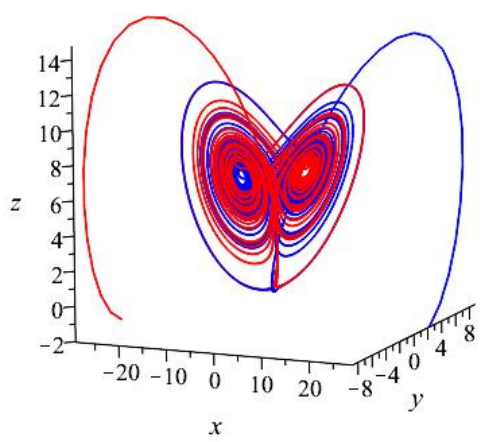

Figure 12 Two solutions of Rabinovich system (25) with parameter values $h=6.75, v=1, v_{1}=3.8$ and initial conditions $( \pm 0.6, \pm 0.6,0)$.

As the invariant algebraic planes $-x^{2}+z^{2}=0$ does not depend on the variable $y$, we can modify the second equation of the system without changing the invariant algebraic surface, hence based on our knowledge on the existence of the four-wings attractor shown in Figure 13, we will take the parameter values $v_{1}=v_{3}=-1.5$ and $v_{2}=0.3$. In this case, system (15) has five singular points belonging to the invariant algebraic surface $-x^{2}+z^{2}=0$ : four unstable foci and one saddle at the origin (see Figure 14). Hence, the global two-dimensional unstable manifolds of the unstable foci are contained in the invariant planes $-x^{2}+z^{2}=0$ and the global one-dimensional unstable manifold of the saddle at the origin is given exactly by the intersection of these planes.

Keeping $h=0.0$, taking the parameter $v_{1}=-1.5$ and changing slightly the parameter $v_{3}$ to $v_{3}=-1.6$, we can see that system (15) has no longer the two invariant planes, but it is not yet chaotic, see Figure 15. The same occurs for $v_{3}=-1.67$, as shown in Figure 16. In Figures 15 and 16, we have taken the initial conditions $(0.6,1.5,0.6),(-0.6,1.5,-0.6),(-0.6,-1.5,0.6)$ and $(0.6,-1.5,-0.6)$.

In order to deform the two invariant planes with the structure contained on them (five singular points), to generate the chaotic behavior, we will now vary the parameter $h$. Taking $h \in[0,0.01]$ we obtain solutions topologically equivalent to 

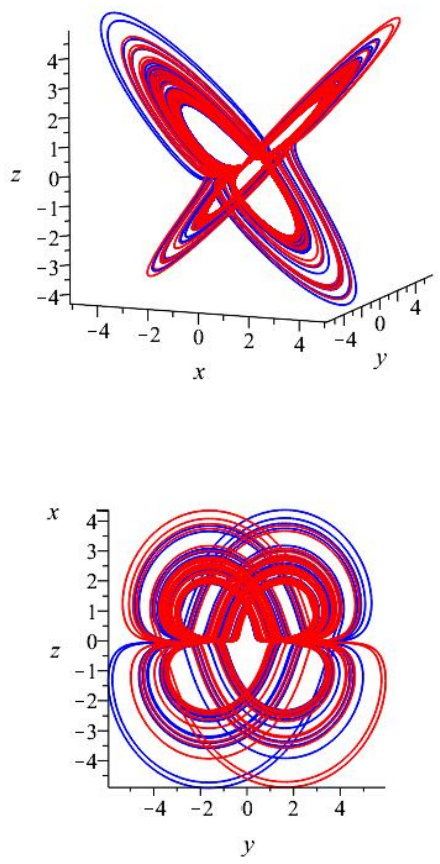

Figure 13 Four-wings chaotic attractor of Rabinovich system with parameters $h=0.04, v_{1}=-1.5, v_{2}=0.3, v_{3}=$ -1.67 , and its projection on the $y z$-plane. Initial conditions: $( \pm 0.6, \pm 1.5, \pm 0.6)$. Time integration: $t \in[800,1100]$.

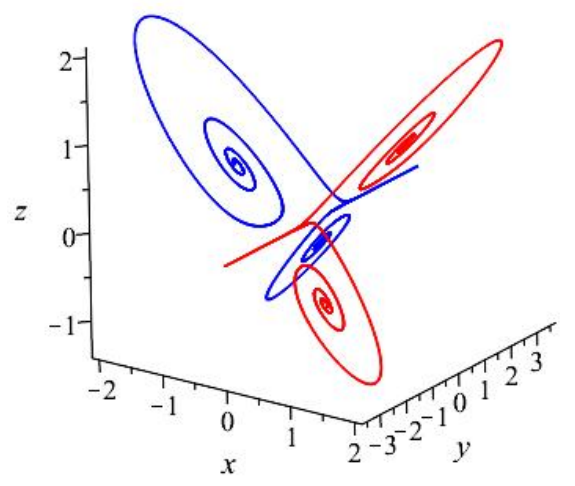

Figure 14 Phase portrait of the system (15) with parameters $h=0.0, v_{1}=v_{3}=-1.5$, and $v_{2}=0.3$. The system has two invariant planes and five equilibrium points: a saddle at the origin and four unstable foci. Initial conditions $(0.6,1.5,0.6),(-0.6,1.5,-0.6),(-0.6,-1.5,0.6)$ and $(0.6,-1.5,-0.6)$.

the ones shown in Figure 17, where we have taken the same initial conditions $(0.6,1.5,0.6),(-0.6,1.5,-0.6),(-0.6,-1.5,0.6)$ and $(0.6,-1.5,-0.6)$ and the time of integration $t \in[300,350]$ in order to exclude the transient part of the solutions and to obtain only the representation of the $\omega$-limit set.

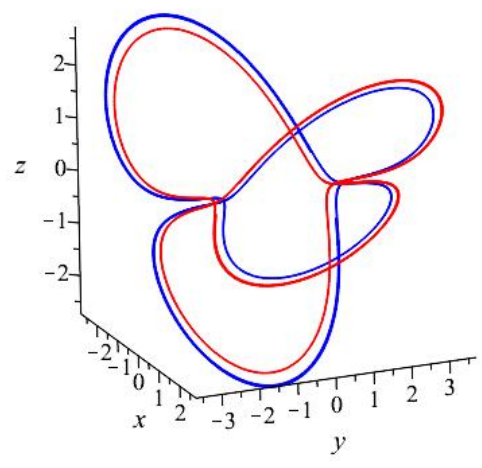

Figure 15 Phase portrait of system (15) with parameters $h=$ $0.0, v_{1}=-1.5, v_{3}=-1.6, v_{2}=0.3$ The system has no longer the invariant planes, but there is no chaotic behavior yet. Time integration: $t \in[120,150]$.

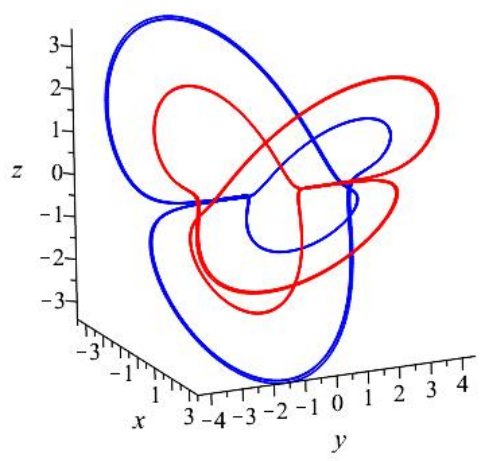

Figure 16 Phase portrait of system (15) with parameters $h=$ $0.0, v_{1}=-1.5, v_{3}=-1.67, v_{2}=0.3$ Time integration: $t \in$ $[120,150]$.

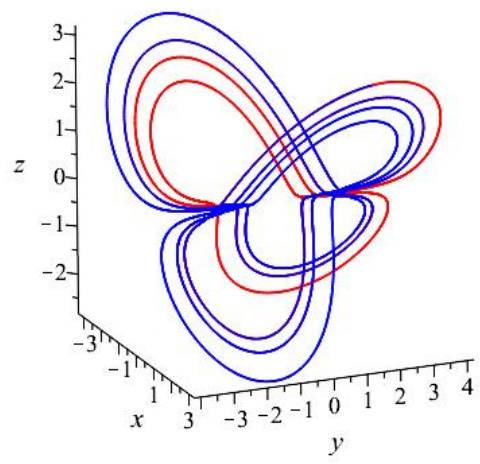

Figure 17 Phase portrait of system (15) with parameters $h=$ $0.01, v_{1}=-1.5, v_{3}=-1.67, v_{2}=0.3$ Time integration: $t \in[300,350]$.

Taking $h=0.015$ and the same initial conditions, we observe that the solutions become more complex, as shown in Figure 18.

Now taking $h \in[0,0.02]$ and the same initial conditions, we 


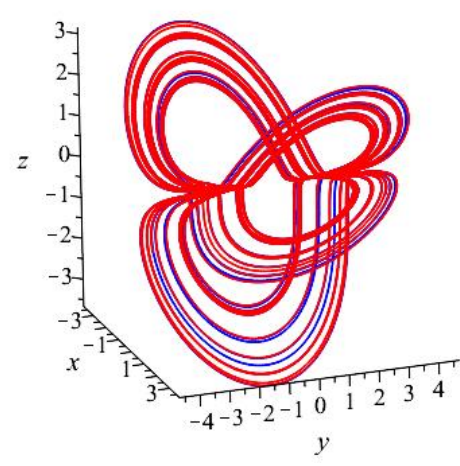

Figure 18 Phase portrait of system (15) with parameters $h=$ $0.015, v_{1}=-1.5, v_{3}=-1.67, v_{2}=0.3$ Time integration: $t \in[600,800]$.

finally obtain a four-wings chaotic attractor, shown in Figure 19, which is similar to the attractor of Figure 13.

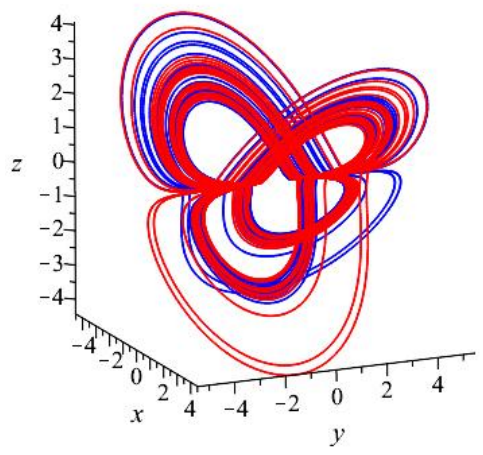

Figure 19 Phase portrait of system (15) with $h=0.02, v_{1}=$ $-1.5, v_{3}=-1.67, v_{2}=0.3$ Observe the existence of a four-wings chaotic attractor. Time integration: $t \in[800,1000]$.

We can see from Figures 14 to 19 that the deformation of the invariant manifolds of the equilibrium points of system (15), which are contained on the invariant planes for $h=0.0$ and $v_{1}=v_{3}$, through the variation of the parameter values, lead to the transition from nonchaotic to chaotic behavior of this system.

\section{CONCLUSIONS}

In this paper we gave sufficient algebraic conditions for some classes of the generalized Lorenz-like system (3) to be nonchaotic. More precisely, these systems have no chaotic behavior when they have an invariant algebraic surface with constant cofactor, as stated in Theorem 1. We also have shown that, in some cases, the deformation of the invariant manifolds of equilibrium points, contained on the invariant surfaces of nonchaotic Lorenz-like systems, by perturbing their parameter values, can deform and reorganize the global phase space structure, leading to the chaotic behavior of these systems. The results presented here are quite general and can be used to study other Lorenz-like systems than the examples presented here.

We believe that the results presented here are somehow related to the works (Osinga and Krauskopf 2002, 2004) on the determination of global one or two-dimensional stable and unstable manifolds of critical elements (mainly singular points and periodic orbits) of chaotic differential systems (as the Lorenz system), in order to describe how these manifolds organize the global phase space of such systems. In these studies, a better understanding of the global behavior of chaotic dynamics were obtained. Analogously, in this paper we could see that the invariant algebraic surfaces of nonchaotic Lorenz-like systems contain the stable (or unstable) manifolds of critical elements because, as the cofactor of these surfaces are constant, all the equilibrium points of the nonchaotic systems are contained on them. Also, the deformation of these invariant algebraic surfaces may lead do the creation of chaotic dynamics, as shown for instance in the Rabinovich system. We think that this ideas may be further developed, aiming to obtain a better understanding of the organization of phase space for nonchaotic and chaotic differential systems by the stable and unstable manifolds of their equilibrium points.

We can conclude saying that, in order to have a better understanding of the complex dynamical behavior of continuous threedimensional differential systems, it is important to study also the nonchaotic differential systems, beyond the chaotic ones.

\section{Acknowledgements}

The first author was supported by State of São Paulo Foundation (FAPESP) grant number 2019/10269-3 and by CNPq/Brazil grant number 311355/2018-8. The second author was supported by CAPES/Brazil through a PhD fellowship.

\section{Conflicts of Interest}

The authors declare that there is no conflict of interest regarding the publication of this paper.

\section{Availability of data and material}

Not applicable.

\section{LITERATURE CITED}

Algaba, A., Dominguez-Moreno, M. C., Merino, M. and RodríguezLuis, A. J., 2018 A Review on Some Bifurcations in the Lorenz System. Nonlinear Systems, 1:3-36.

Alligood, K. T., Sauer, T. and Yorke, J., 1996 Chaos: An Introduction to Dynamical Systems. Springer-Verlag, New York.

Anastassiou, S., Pnevmatikos, S. and Bountis T, 2002 Quadratic Vector Fields Equivariant Under the $D_{2}$ Symmetry Group. Internat. J. Bifur. Chaos Appl. Sci. Engrg. 23, 1350017.

Argyris, J., Faust, G., Haase, M. and Friedrich, R., 2015 An Exploration of Dynamical Systems and Chaos. Springer-Verlag, Berlin.

Cencini, M., Cecconi, F. and Vulpiani, A., 2010 Chaos: From Simple Models to Complex Systems. World Scientific, Singapore.

Chen, G. and Ueta, T., 1999 Yet another chaotic attractor. Internat. J. Bifur. Chaos Appl. Sci. Engrg. 9:1465-1466.

Dumortier, F., Llibre, J. and Artés, J.C., 2006 Qualitative Theory of Planar Differential Systems. Springer-Verlag, New York.

Guckenheimer, J. and Holmes, P. [2002] "Nonlinear Oscillations, Dynamical Systems and Bifurcations of Vector Fields", (Appl. Math. Sci. 42, Springer-Verlag, New York).

Heidel, J. and Zhang, F., 1999 Nonchaotic behavior in threedimensional quadratic systems II. The conservative case. Nonlinearity 12:617-633. 
Heidel, J. and Zhang, F., 2007 Nonchaotic and chaotic behaviour in three-dimensional quadratic systems: Five-one conservative cases. Internat. J. Bifur. Chaos Appl. Sci. Engrg. 17:2049-2072.

Jafari, S., Sprott, J. C., Pham, V-T, Volos, C., and Li, C., 2016 Simple chaotic 3D flows with surfaces of equilibria. Nonlinear Dynamics 86:1349-1358.

Li, C., Peng, Y., Tao, Z., Sprott, J. C. and Jafari, S., 2021 Coexisting Infinite Equilibria and Chaos. Internat. J. Bifur. Chaos Appl. Sci. Engrg. 31(5), 2130014, 17p.

Llibre, J., 2004 Integrability of polynomial differential systems. Handbook of differential equations, Elsevier/North-Holland, Amsterdam.

Llibre, J. and Messias, M., 2009 Global dynamics of the Rikitake system. Phys D: Nonlinear Phenomena 238:241-252.

Llibre, J., Messias, M. and da Silva, P.R., 2008 On the global dynamics of the Rabinovich system. J. Phys. A: Math. Theor. 41, 275210, $21 \mathrm{p}$.

Llibre, J., Messias, M. and da Silva, P.R., 2010 Global dynamics of the Lorenz system with invariant algebraic surfaces. Internat. J. Bifur. Chaos Appl. Sci. Engrg. 20:3137-3155.

Llibre, J., Messias, M. and da Silva, P.R., 2012 Global dynamics in the Poincaré ball of the Chen system having invariant algebraic surfaces. Internat. J. Bifur. Chaos Appl. Sci. Engrg. 22, 1250154, $17 p$.

Llibre, J. and Oliveira, R. D. S., 2015 Quadratic systems with invariant straight lines with total multiplicity two having Darboux invariants. Comm. in Contemporary Math. 17, 1450018.

Llibre, J. and Zhang, X., 2012 On the Darboux integrability of the polynomial differential systems. Qualit. Th. Dyn. Sys. 11:129 144.

Lorenz, E.N., 1963 Deterministic nonperiodic flow. J. Atmos. Sci. 20:130-141.

Malasoma, J. -M., 2002 A new class of minimal chaotic flows equation for continuous chaos. Phys. Lett. A. 305:52-58.

Malasoma, J. -M., 2009 Non-chaotic behavior for a class of quadratic jerk equations. Chaos, Solitons Fractals 39:533-539.

Messias, M. and Silva, R. P., 2018 Nonchaotic Behavior in Quadratic Three-Dimensional Differential Systems wiht a Symmetric Jacobian Matrix. Internat. J. Bifur. Chaos Appl. Sci. Engrg. 28, 1830006.
Messias, M. and Silva, R. P., 2020 Determination of nonchaotic behavior for some classes of polynomial jerk equations. Internat. J. Bifur. Chaos Appl. Sci. Engrg. 30, 2050117, 12p.

Osinga, H. M. and Krauskopf, B., 2002 Visualizing the structure of chaos in the Lorenz system. Computers and Graphics 26(5):815823.

Osinga, H. M. and Krauskopf, B., 2004 The Lorenz manifold as a collection of geodesic level sets. Nonlinearity $17 \mathrm{C} 1$.

Ott, E., 2002 Chaos in Dynamical Systems. Cambridge University Press, London.

Rössler, O. E., 1976 An equation for continuous chaos. Phys. Lett. A 57:397-398.

Sparrow, C., 1982 The Lorenz Equations. Bifurcations, Chaos, and Strange Attractors. Springer-Verlag, New York.

Strogatz, S.H., 2001 Nonlinear Dynamics and Chaos: with applications to physics, biology, chemistry and engineering. Westview Press, New York.

Zhang, F. and Heidel, J., 1997 Nonchaotic behaviour in threedimensional quadratic systems. Nonlinearity 10:1289-1303.

Zhang, F. and Heidel, J., 2012 Chaotic and nonchaotic behaviour in three-dimensional quadratic systems: 5-1 dissipative cases. Internat. J. Bifur. Chaos Appl. Sci. Engrg. 22, 1250010.

Zhang, F., Heidel, J. and Le Borne, R., 2008 Determining nonchaotic parameter regions in some simple chaotic jerk functions. Chaos, Solitons and Fractals 11:1413-1418.

Zhu C., Liu Y. and Guo Y., 2010 Theoretic and Numerical Study of a New Chaotic System. Intelligent Information Management 2:104-109.

Yang, X. S., 2000 Nonchaotic behavior in nondissipative quadratic systems. Chaos, Solitons and Fractals 11:1799-1802.

Yang, X. S., 2002 On non-chaotic behavior of a class of jerk systems. Far East J. Dyn. Syst. 4:27-38.

Yang, X. S. and Chen, G., 2002 Non-chaotic behavior in a class of continuous dynamical systems. Far East J. Dyn. Syst. 4:87-95.

Wiggins, S., 2003 Introduction to Applied Nonlinear Dynamical Systems and Chaos. Texts in Appl. Math. 2, Springer-Verlag, New York.

How to cite this article: Messias, M. and Silva, R. P. Nonchaotic Behavior and Transition to Chaos in Lorenz-like Systems Having Invariant Algebraic Surfaces. Chaos Theory and Applications, 4(1), 26-36, 2022. 